\title{
Formación del profesorado en la lengua y cultura indígena: una histórica demanda educativa en contexto Mapuche'
}

Omar Turrall

Donatila Ferrada"I

I- El presente artículo comunica resultados del proyecto Fondecyt Regular № 1151233 y No1140363.

II- Universidad del Bio Bio, Chillán, Chile.

Contacto: oturra@ubiobio.cl

III- Universidad Católica del Maule, Talca, Chile.

Contacto: donatila.ferrada@gmail.com

\section{Resumen}

Este artículo presenta resultados de una investigación que tuvo como propósito construir, junto al profesorado en ejercicio en contexto educativo y cultural Mapuche, los requerimientos que estos docentes demandan a las instituciones formadoras en función de las necesidades del contexto de desempeño. Los requerimientos actuales del profesorado se correlacionan, en perspectiva histórica, con las demandas levantadas por un grupo de profesores pertenecientes a este pueblo originario y testimoniado hacia los años 30 y 40 del siglo XX. La investigación se desarrolló desde una metodología de investigación social mixta, de carácter histórico para concentrar las demandas del profesorado Mapuche y desde la metodología comunicativa para levantar las demandas actuales del profesorado. Para este último caso también se implementó un diseño de investigación mixto, de tipología secuencial e integrada que utilizó distintas técnicas de recolección de información durante su desarrollo (cuestionario comunicativo, entrevista comunicativa y grupo de discusión comunicativo). Los resultados de ambos corpus de requerimientos confluyen en demandar un profesorado con formación en la lengua y cultura Mapuche, que le permita dar cuenta de las características culturales y lingüísticas del estudiantado originario que asiste al sistema escolar, es decir abogan por la presencia de un cuerpo docente con habilitación profesional para desarrollar procesos pedagógicos con pertinencia cultural.

\section{Palabras clave}

Formación de profesores - Lengua y cultura indígena - Contexto Mapuche - Profesorado Mapuche - Mapuzugun. 


\section{Teacher education in indigenous language and culture: A historical educational demand in Mapuche context}

Omar Turra"

Donatila Ferradall'

I- This article is part of the investigation Proyecto Fondecyt Regular No 1151233 y N ${ }^{\circ} 1140363$

II- Universidad del Bio Bio, Chillán, Chile. Contact: oturra@ubiobio.cl

III- Universidad Católica del Maule, Talca, Chile.

Contact: donatila.ferrada@gmail.com

\begin{abstract}
This article brings the results of a study whose purpose was to construct with teachers working in a Mapuche cultural and educational context what they demand from educational institutions according to the needs for their performance. Teachers' current requirements are correlated, in a historical perspective, with the demands of a group of teachers from this indigenous people witnessed in the 1930s and 1940s. The study has been developed with a mixed social research methodology of historical character to focus on the Mapuche teachers' demands, and with a communicative methodology to collect current teachers' demands. For the latter, a mixed research model with a sequential integrated typology was implemented, in which different data collecting techniques were used (communicative questionnaires, interviews, and discussion groups). The results of both corpora of requirements converge on the need for teachers with training in Mapuche culture and language, so that they can deal with the cultural and linguistic characteristics of Mapuche students. In other words, they demand teachers be qualified to develop culturally relevant education.
\end{abstract}

\section{Keywords}

Teacher education - Indigenous language and culture - Mapuche Mapuche teachers - Mapuzugun. 


\section{Introducción}

Al presente, existe consenso en Chile que la escolarización en relación con los pueblos originarios debe asumir nuevas perspectivas y propósitos, considerando la pauperización cultural y exclusión social que históricamente la escuela ha generado entre ellos. Predomina, a nivel político e intelectual, un discurso educativo de base multiculturalista que reconoce la alteridad indígena y sus derechos educativos y confía en el rol privilegiado de la escuela en la construcción de nuevos sentidos formativos que permitan revalorizar el conocimiento, la lengua y toda manifestación cultural de los pueblos originarios.

En el marco del reconocimiento de la deuda histórica que el Estado tiene con los pueblos originarios se ha impulsado, desde los años noventa, la educación intercultural bilingüe como un programa educativo compensatorio y focalizado exclusivamente en el estudiantado indígena, como una forma de contrarrestar el debilitamiento de la identidad étnica y el rezago educativo que caracteriza a estas comunidades. Profundizando en esta línea de acción, recientemente se ha incorporado al currículum escolar la asignatura de Lengua Indígena.

Sin embargo, estas iniciativas de política pública educativa se han promovido en desvinculación con los procesos de formación inicial de profesores que se han implementado en el país, que no han considerado la dimensión cultural y lingüística indígena en sus programas. Es decir, se carece de un cuerpo docente que permita efectivamente dar cuenta de los propósitos de la política y de atención especializada al universo de estudiantes indígenas que asisten en la actualidad al sistema escolar. Este problema de formación del profesorado quedó en evidencia en el trabajo investigativo con docentes primarios en contexto mapuchelafkenche (Tirúa) y mapuche-pehuenche (Alto Biobío), quienes en el proceso de recuperación de sus requerimientos de formación inicial para un desempeño profesional de calidad, configuraron como demanda una formación en la lengua y cultura indígena.
El trabajo cotidiano de estos profesores con niños y niñas provenientes de comunidades indígenas, muchos/as de ellos/as solo hablantes de la lengua materna (mapuzugun) al momento de ingresar al sistema escolar, coloca de manifiesto la diversidad sociocultural del alumnado y la necesaria formación del profesorado en lenguaje y comunicación para la diversidad lingüística y no sólo en lengua castellana.

Un particular hallazgo al respecto, fue la constatación de que esta demanda educativa se encuentra documentada desde la primera parte del siglo XX, específicamente entre los años 30 y 40 del siglo pasado, y que sus impulsores fueron profesores mapuches que a la época levantaron sus voces por una educación con pertinencia cultural y lingüística y una formación docente en consecuencia. Es de interés, entonces, exponer en este artículo el contenido de estas demandas, en perspectiva histórica, pues permite generar comprensión acerca de los procesos educativos en contexto mapuche y los desafíos de una formación docente para un desempeño bilingüe e intercultural.

\section{Marco teórico}

\section{Antecedentes. Programas formativos en territorio Mapuche}

En el extenso territorio mapuche, en la Araucanía según denominación castellana ${ }^{1}$, las relaciones coloniales configuraron dos modelos de educación con fines específicos y diferenciados cuyas rutas formativas se pueden rastrear hasta la actualidad. Son programas formativos que se desarrollaron de manera paralela en un contexto de relaciones interétnicas. ${ }^{2}$ Para el caso de la sociedad mapuche, el programa educativo organiza el conocimiento, los saberes educativos a transmitir de acuerdo a las edades y el género y las formas

1- Los conquistadores españoles denominaron araucanos al pueblo mapuche, y desde entonces este concepto es resistido por su impronta colonialista.

2- Son los cronistas españoles del siglo XVII y XVIII quienes testimoniaron la existencia de un conjunto de saberes, conocimientos y destrezas como constituyentes de un itinerario formativo de los infantes y jóvenes mapuches, así como unas formas de enseñar y aprender que se diferenciaban según el género y la edad. 
de enseñar y aprender en la comunidad, es decir orienta el proceso de formación de persona que la sociedad mapuche construyó para formar a sus nuevas generaciones y garantizar su proyección como sociedad y como pueblo (CATRIQUIR; DURÁN, 1997, 2007). Es un programa educativo sin escuela -en el entendido que esta institución es de origen occidental- pero que da cuenta del horizonte conceptual y principios educativos que organizaban el proceso de enseñanza y aprendizaje en la sociedad mapuche.

Por otra parte, el asentamiento español en los márgenes de la Araucanía desde los tiempos de la conquista, señala el inicio del modelo educacional occidental, basado en la enseñanza de la lectura y escritura castellana entre las comunidades mapuche. Este modelo educacional se inscribió en la estrategia de evangelización que estaba a la base de la conquista española en América, por lo que fue desarrollado por sacerdotes pertenecientes a congregaciones religiosas que llegaron con los primeros conquistadores (PINTO, 1990). Este programa formativo buscaba la asimilación y/o aculturación de los jóvenes mapuche a la cultura hispanooccidental, por lo que se sintetizaba en la iniciación de los educandos indígenas en la doctrina católica y en la castellanización de su lenguaje.

Luego del proceso independentista el Estado chileno replicó el modelo de las escuelas misionales coloniales en la Araucanía, siendo la evangelización y castellanización la base del programa formativo pero ahora con el propósito de integrar al pueblo Mapuche a la civilización y a la chilenidad.

Con regularidades y variaciones los programas formativos mantuvieron sus líneas de continuidad mientras la sociedad mapuche e hispano-criolla se desarrollaban en base a relaciones interétnicas coloniales, pero en que ambas se movilizaban desde su soberanía cultural y política.

Sin embargo, esta situación de convivencia interétnica y de proyección de los programas formativos experimentó un drástico revés histórico, hacia fines de la centuria decimonónica, cuando las formas culturales del pueblo mapuche fueron sometidas a un proceso de degradación en el imaginario colectivo de la chilenidad, hasta ser considerados como verdaderos obstáculos para el proceso modernizador impulsado por el Estado. Se configuró un discurso orientado a legitimar la ocupación de sus tierras y exclusión del proyecto sociocultural que se aspiraba a conformar (MARTÍNEZ, 1991; PINTO, 1992, 2000). Esta configuración discursiva se expresó materialmente en la ocupación de la Araucanía (1880-1883) y el consecuente proceso de reducción de la población mapuche en tierras destinadas para el efecto (BENGOA, 2000).

Esta coyuntura histórica constituye un hito fundamental en la relación del Estado chileno con el pueblo Mapuche, cuyas implicancias se proyectan hasta la actualidad. El Estado nacional definió un problema central de interés económico y geopolítico, como es la integración territorial de la Araucanía; para la sociedad Mapuche significó la pérdida de su autonomía política-cultural y el comienzo de un proceso de pauperización y forzada aculturación.

Las nuevas condiciones generadas por la ocupación del territorio y despojo de tierras, con la pérdida de su soberanía como pueblo originario se produce un repliegue del programa formativo Mapuche a una esfera privada, al espacio familiar inmediato, configurándose como una instancia de oposición y/o resistencia al modelo educativo oficial representado por la escuela chilena (MARIMÁN, 1997; CANALES, 1998). También se ha testimoniado la actitud de resistencia hacia la escuela chilena por parte de sectores y comunidades aferrados a los patrones culturales ancestrales, pues la enseñanza escolar se asociaba a la imposición de normas y hábitos foráneos. Desde estas voces, la escuela era vista como un espacio de perdición del ser Mapuche $\mathrm{y}$ por lo tanto de necesaria impugnación $\mathrm{y}$ repudio (MARIMÁN, 1997).

En este contexto, la política educacional del Estado chileno en la Araucanía tuvo como eje central consolidar la instrucción pública en los pueblos que se iban fundando, creando escuelas en los poblados mayores para desde ahí extenderlas hacia aldeas y pequeños villorrios (SERRANO, 
1995-96). Una educación orientada hacia el mundo rural, propiamente tal, estaba de plano descartado al igual que una educación específica a mapuches en sus nuevos espacios de reducción. Por tanto, la posibilidad de acceder al aprendizaje de la lectura y escritura quedó reducida a un grupo minoritario de educandos mapuches que asistían a la escuela chilena en los pueblos.

\section{Formación del profesorado y pueblos originarios}

La investigación especializada en formación docente ha planteado el rol preponderante del profesorado al momento de pensar cualquier transformación educativa, considerando su rol clave para el logro de aprendizajes en el estudiantado (FULLAN, 2002; AGUERRONDO, 2004; VAILLANT, 2005). Sin embargo, también se ha constatado que los programas de formación inicial de profesores presentan una escasa reflexión respecto del sentido de la enseñanza en los actuales escenarios de escolaridad y una insuficiente preparación para trabajar en contextos socioculturales diversos (VEZUB, 2007). En esta línea argumentativa, se encuentran aquellos estudios que describen el desencuentro entre la formación inicial recibida y la realidad profesional presente en la diversidad de contextos escolares de los sistemas educativos (BUSTOS, 2007; GONZÁLEZ-VALLINAS; OTERINO; SAN FABIÁN, 2007).

Por otro lado, se plantea la necesidad de garantizar la pertinencia cultural de los procesos pedagógicos (TORRES, 2008; DÍAZ-BARRIGA, 2005; BESALÚ, 2002) con el propósito de valorizar y reforzar las identidades culturas locales. Aquello involucra avanzar hacia un entendimiento de la escuela en estrecha vinculación con las comunidades sociales y los territorios en que se encuentra, en donde la organización pedagógica-curricular de cuenta de las formas culturales locales, para aportar a la autovaloración de la propia identidad y desde ahí proyectarse en el conocimiento y valoración de las diferencias culturales en contextos más amplios. Partir de la cultura de referencia para ampliarla a otras favorece a la igualdad educativa, pues se sustenta en aprendizajes expansivos que aseguran al estudiantado un desenvolvimiento en la sociedad de un modo diferente a como lo habrían hecho solo con la cultura de que disponen (SEPÚLVEDA, 2001).

En América Latina, los discursos educativos de reconocimiento de la diversidad sociocultural han devenido en la implementación de programas específicos orientados a la revitalización de las lenguas indígenas a través del sistema escolar, en el contexto del reconocimiento de los derechos educativos y culturales de los pueblos originarios. Sin embargo, la investigación comparada (LÓPEZ; SICHRE, 2004; WILLIAMSON, 2004) evidencia la variedad conceptual que asume esta respuesta educativa en los distintos estados, denominándose en algunos casos Educación Multicultural, Educación Indígena, Etnoeducación, Educación Intercultural y, de manera predominante, Educación Intercultural Bilingüe, cuestión que refleja la diversidad de entendimientos que está a su base y las tensiones y contradicciones respecto de sus alcances al interior de los propias administraciones educativas.

Solo recientemente, la formación docente y sus actuaciones en contextos interétnicos e interculturales se visualizan como un eje problemático para la investigación educativa. Así, para el caso mexicano surgen estudios que focalizan su interés en las trayectorias profesionales de docentes indígenas (RAYÓN, 2004), en el reconocimiento de los procesos de formación docente recibida por estos profesores, para alcanzar comprensión acerca de la transmisión de valores nacionales-estatales y los propios de la cultura indígena (LÓPEZ, 2005). También se ha estudiado la formación profesional de docentes que se desempeñan en la educación bilingüe, quedando en evidencia la insuficiente preparación y actualización de los docentes en general y bilingües en particular, de una formación en perspectiva intercultural (HERNÁNDEZ, 2008).

Otra investigación, situada en contexto colombiano, describe el tipo de formación y capacitación en etnoeducación recibida por los docentes indígenas Ingas y constata la distancia entre los objetivos del programa en 
etnoeducación y los propósitos formativos demandados por los docentes originarios (CARLOSAMA, 2001).

Para el caso de Chile, la formación inicial del profesorado se organiza en base a un desempeño homogéneo, es decir, prepara para atender pedagógicamente a un estudiante promedio a la base de un currículo monocultural de cuño europeo. Con ello se estandariza la diversidad de desempeños a los cuales podría enfrentarse el futuro profesorado, omitiendo la diversidad de territorios, cosmovisiones, comprensiones culturales, es decir una formación pedagógica en interculturalidad (MANZO; WESTERHOUT, 2003). También se ha omitido en los procesos de renovación del currículo de formación inicial, las voces de sus protagonistas principales, el propio profesorado en ejercicio laboral (FERRADA; TURRA, 2012). Con ello, escasamente se favorece el protagonismo docente para el cambio educativo, por el contrario se contribuye a sostener sistemas educativos jerárquicos y cerrados (ROBALINO, 2005).

Desde la política educativa para pueblos originarios se plantea el manifiesto propósito de preservar la cultura y la lengua de estos pueblos, a través de transformaciones curriculares que incorporan elementos propios de la cultura indígena en los procesos formativos del sistema escolar (Programa de Educación Intercultural Bilingüe y asignatura Lengua Indígena en el currículo de Educación Básica). Sin embargo, estas definiciones de política pública no se han traducido en transformaciones en el currículo de formación de profesores que recoja las especificidades culturales y laborales de los contextos de indigeneidad (TURRA; FERRADA; VILLENA, 2013). Así, los profesores que se desempeñan laboralmente en contextos educativos de pueblos originarios entran en contacto con lógicas culturales y cosmovisiones diferenciadas, además de especificidades curriculares y pedagógicas, aspectos todos que los llevan a asumir un desempeño profesional para el cual no han sido preparado (QUINTRIQUEO, 2010).

Por el contrario, una formación del profesorado pensada desde la consideración de los contextos culturales implica romper con la formación uniforme y avanzar hacia el diseño de currículos transformadores, que diversifiquen sus procesos de formación en consideración a la diversidad cultural y lingüística en que se desarrolla la acción educativa (FERRADA; TURRA; VILLENA, 2013).

\section{Metodología}

La investigación se desarrolló desde una metodología de investigación social mixta, por cuanto el objeto de estudio se abordó en perspectiva histórica, considerando dos momentos específicos de su desarrollo como fenómeno social. El primero, define las demandas docentes a través de la prensa de la Araucanía entre los años 1935-1946, por medio de una investigación de carácter histórico; el segundo, define las demandas docentes expresadas en el marco de un proyecto de investigación educativa desarrollado entre los años 2011-2013. No obstante, ambas expresiones de demandas de formación docente se sitúan espacialmente en el tradicional territorio mapuche, conocido como la Araucanía; para el primero, los testimonios se vinculan al entorno de la ciudad de Temuco, el centro territorial definido por el estado chileno; para el segundo, la investigación trabajó con docentes pertenecientes a la Provincia de Arauco y del Biobío, ambos espacios territoriales localizados en las márgenes norte de la Araucanía.

Para el período correspondiente al siglo XX, nos servimos del estudio documental de Foerster y Montecino - Organizaciones, líderes $y$ contiendas mapuches (1900-1970) - (1988), que, utilizando como fuente principal la prensa local, el Diario Austral de Temuco, expone diversos ámbitos de demanda hacia el estado chileno por parte de la sociedad mapuche. Entre ellas, se identifica un corpus de demandas de formación de profesores para el contexto mapuche, expresadas por un grupo de docentes pertenecientes al propio pueblo originario. De los documentos de época se seleccionaron testimonios y declaraciones que aportan a los objetivos del estudio, y que permitieron configurar las categorías de demandas levantadas. 
Para el caso de la investigación con el profesorado de Arauco y Biobío, se utilizó la metodología comunicativa de investigación (GÓMEZ et al., 2006). En su conformación como paradigma de investigación, la metodología comunicativa presenta una fundamentación arraigada en dimensiones ontológica, epistemológica y metodológica, que permite distinguirla y diferenciarla de otras corrientes de investigación, fundamentos que se exponen en diversos documentos de circulación científica (GÓMEZ; RACIONERO, 2008; GÓMEZ; DIEZPALOMAR, 2009; GÓMEZ; HOLFORD, 2010; FERRADA, 2011). A su vez, reporta técnicas propias de recogida y de análisis de información de carácter comunicativo, como también cuenta con criterios de rigor específicos que permiten validar la información recogida (GÓMEZ et al., 2006).

En términos metodológicos, al igual que las otras corrientes de investigación, tiene como propósito describir y explicar la realidad, comprenderla e interpretarla, pero se diferencia de ellas al proponerse a estudiar la realidad social para transformarla, mediante el diálogo y reflexión conjunta que permita alcanzar comprensión respecto de los procesos que generan la exclusión o inclusión social. Al fundarse en una ontología que entiende la realidad como producto de significados que se construyen comunicativamente mediante la interacción entre las personas, se sostiene que solo es posible construir el objeto de estudio a través de las interpretaciones, reflexiones y teorías de las propias personas participantes en la realidad social que se quiere transformar (GÓMEZ et al., 2006). Desde un nivel ontológico, la metodología comunicativa considera que existe una realidad natural/objetiva con independencia de los sujetos y una realidad que se construye por medio de las interacciones de sujetos capaces de lenguaje y acción, y por lo tanto dependiente de los sujetos. Esta doble dimensión, permite a nivel epistémico y metodológico, la generación de conocimiento cuantitativo y cualitativo, a base de la racionalidad comunicativa.

En esta perspectiva, la investigación asumió un diseño mixto, pues consideró fases cuantitativas y cualitativas, con un carácter secuencial e integrado. La secuencialidad considera un diseño cuantitativo o cualitativo primero, para luego desarrollar el otro (CRESWELL, 2009), y el diseño integrado, considera la integración de ambos métodos durante todo el proceso investigativo, representando el más alto grado de combinación entre lo cualitativo y cuantitativo (CRESWELL, 2009; HERNÁNDEZ; FERNÁNDEZ; BAPTISTA, 2010). Por tanto, la secuencialidad e integración fue transversal en la investigación, ya que un diseño dependía de los datos arrojados por el anterior, al igual que las muestras, en que los sujetos que participaron en un método lo volvieron a hacer en el otro, y finalmente, las técnicas de recolección de la información usadas reportaron los datos para la selección de la siguiente técnica.

Se trabajó con una muestra representativa de profesores de educación básica que se desempeñan en escuelas públicas (municipales), caracterizadas como de alta vulnerabilidad escolar (sobre 80 IVE, Índice de Vulnerabilidad Escolar) de ambas provincias, a la base de los criterios siguientes: 1) que realicen docencia en el primer ciclo básico; 2) que tengan una experiencia profesional de diez años como mínimo en el contexto; 3) que adquieran el compromiso de participar voluntariamente en la investigación. De acuerdo a estos criterios, durante el proceso investigativo participaron 82 profesores, correspondiendo 46 a la provincia de Biobío y 36 a la de Arauco, con un promedio de experiencia docente de veintiséis años.

Las técnicas de recogida de información fueron el cuestionario comunicativo, la entrevista comunicativa y el grupo de discusión comunicativo. El cuestionario comunicativo es un conjunto de preguntas (abiertas o cerradas) respecto de una o más variables a medir, construidas con y en acuerdo con los propios sujetos investigados. Por lo mismo, requiere de una técnica cualitativa de orientación comunicativa previa para su elaboración que puede ser el grupo de discusión o la entrevista comunicativa (FERRADA, 2011), para el caso de esta investigación se utilizó el grupo de discusión comunicativo, y se construyó un cuestionario 
dicotómico. La entrevista comunicativa, comprende una conversación entre una persona investigadora y una investigada, mediante la cual se pretende llegar a acuerdos respecto de un tema de interés común (PÁDROS et al., 2011), conversación que admite discrepancias, debates y cuestionamientos que se resuelven sobre la base del mejor argumento mediados por pretensiones de validez. Por su parte, el grupo de discusión comunicativo, supone un diálogo igualitario entre varias personas pertenecientes al grupo o comunidad objeto de investigación y otra persona que forma parte del equipo investigador. A través de este diálogo, se construye una interpretación colectiva del tema en estudio, que recoge el conocimiento científico existente sobre el tema aportado por el equipo investigador y su contraste con el conocimiento social-experiencial aportado por las personas de la comunidad que componen el grupo. Se realiza en un contexto conocido por las personas participantes y además el grupo elegido es natural, está compuesto por personas que ya se conocen y suelen coincidir y juntarse para realizar diversas actividades (AUBERT et al., 2004).

Por su parte, la validación de la información recogida se realizó mediante la aplicación de los tres criterios de rigor de la metodología comunicativa: diálogo intersubjetivo, pretensión de validez y compromiso (GÓMEZ et al, 2006). De este proceso, resultaron las demandas de formación que exponemos en el siguiente apartado.

\section{Resultados}

\section{La demanda actual de formación del profesorado en la lengua indígena}

La investigación con el profesorado consideró como uno de los ámbitos de requerimientos de formación inicial a investigar, desde la experiencia profesional docente en contexto, la formación en lenguaje y comunicación. Para ello, el cuestionario contempló, entre varias preguntas referidas al ámbito, una pregunta que consultaba si era un requerimiento de formación inicial formar al futuro profesorado en la lengua propia del grupo indígena para promover un desempeño contextualizado en la cultura. La respuesta dio como resultado un $80 \%$ de aprobación por parte del profesorado.

Posteriormente, en la fase cualitativa se profundizó en la variable tanto a nivel de entrevista comunicativa como de grupo de discusión, de tal forma que a partir del requerimiento original emergieron otras demandas asociadas que permitieron ampliar y sostener una formación en lenguaje y comunicación en la lengua indígena mapuzugun. Ellas fueron:

- Formar para comprender que el bilingüismo en ambas direcciones es la base de la educación intercultural;

- Formar al futuro profesor para promover aprendizajes en la lengua indígena relacionándolos con otros sectores de aprendizajes;

El contexto de desempeño laboral del profesorado, con una alta matrícula escolar mapuche en los establecimientos educacionales, constituye la base explicativa de estas demandas. Es así como en la Provincia del Biobío, en la zona cordillerana, espacio de ocupación tradicional de la identidad territorial mapuche-pehuenche la lengua originaria se encuentra plenamente vigente, presentándose situaciones en que el estudiantado indígena ingresa al sistema escolar hablando solo la lengua originaria. Un testimonio de una docente de Alto Biobío coloca en evidencia esta situación:

[...] porque los primeros años cuando llegan niñitos que vienen cero castellano, seria buenísimo trabajar con profesores que manejen bien la lengua indígena, porque a nosotros a veces también se nos ha hecho difícil...Entonces hay que formar profesores en la lengua para comunicarnos con ellos...si es que los profesores quieren irse a trabajar a la comunidades que tienen lenguas diferentes, hay que formarlos para eso. (Entrevista comunicativa, profesora de Alto Biobío, 2013).

Respecto de la provincia de Arauco, espacio ocupado por la identidad territorial 
Mapuche-lafkenche, si bien el retroceso de la lengua originaria es constatado por los docentes, existe conciencia entre ellos respecto de la necesidad de legitimar la utilización de la lengua indígena en la escuela, a través de su dominio por parte del profesorado.

Yo pienso que teniendo estudiantes de descendencia del pueblo originario mapuche... no podemos nosotros como coartarlo, o sea ahí lo mejor es que el profesor conozca la lengua, el saber no ocupa espacio dicen, si yo sé más, me enriquezco como persona y profesional. Por lo tanto, yo creo que ahí al profesor habría que capacitarlo para poder compenetrarse $o$ poder entender un poco más de adentro la lengua y cultura mapuche. (Entrevista comunicativa, profesor de Curanilahue, 2013).

Además, desde este territorio se ha impulsado una fuerte reivindicación cultural y lingüística que se ha traducido en medidas de política pública educativa para el sistema escolar en contextos de pueblos originarios, como la educación intercultural bilingüe y recientemente la introducción de la asignatura Lengua Indígena. Sin embargo, todo ello se ha realizado sin una formación continua del profesorado en la lengua originaria, tampoco su incorporación en los programas de formación inicial docente. Ello explica las demandas planteadas desde la dimensión cualitativa.

También, dentro del ámbito de formación del profesorado en la lengua indígena surgieron dos demandas, desde la fase cualitativa, que se asocian directamente con una formación para saber trabajar con las formas y saberes culturales Mapuche en los procesos pedagógicos desarrollados en contextos interétnicos mapuche/ no mapuche. Ellas son:

- Formar para comprender la cultura del alumnado mapuche;

- Formar para comprender que la cultura indígena que porta el estudiantado tiene el mismo estatus que la cultura escolar

Estas demandas solo se pueden satisfacer desde el conocimiento y dominio de la lengua
Mapuche. Lo que se expresa en estas demandas es, primero, una formación docente en aspectos fundamentales que permitan alcanzar comprensión respecto de los fundamentos de la lógica cultural mapuche, sus concepciones de mundo, y su expresión en determinadas formas culturales.

Por su parte, la segunda demanda remite a un trabajo en el sistema escolar en contexto interétnico con saberes educativos mapuche, lo que requiere además una formación en criterios curriculares para equilibrar los saberes a enseñar y otorgar legitimidad como conocimiento escolar a los saberes originarios.

Para el contexto educativo interétnico, se trata de demandas absolutamente pertinentes, pues a nivel de declaraciones educativas se reconoce la diversidad sociocultural en educación y los derechos educativos de los pueblos originarios.

\section{La demanda histórica de formación del profesorado en la lengua indígena}

Hacia 1930, en el antiguo territorio Mapuche ya habían transcurrido cinco décadas de la ocupación (despojo) y control estatal de estas tierras y se expandía la población en ciudades, pueblos y villas, al mismo tiempo que el Estado y sus instituciones. Entre estas últimas, la escuela, institución reconocida por su capacidad de agencia en la promoción de la ideología estatal, ejercía su rol alfabetizador y civilizador en el espacio interétnico. En este contexto es que se configuró la demanda educativa mapuche de la época, como una expresión de resistencia a la violencia simbólica estatal, pero también como un espacio de posibilidad ante las expectativas educativas que el propio estado generaba.

La demanda educativa fue impulsada por un grupo de profesores mapuche descontentos con el servicio educativo estatal para indígenas, que los llevó a levantar sus voces por una educación pública que se hiciera cargo de la especificidad demográfica, cultural y lingüística mapuche. Para dar cuenta de la aspiración por una enseñanza con pertinencia cultural, plantearon la necesidad de formar profesores provenientes del propio pueblo originario y hablantes del mapuzugun. 
$\mathrm{Al}$ respecto, la profesora Zoila Quintremil, en una exposición dirigida a docentes de escuelas de carácter indígena, realizada en la ciudad de Temuco el año 1935, expresaba la necesidad de "fundar colegios internados en el campo mismo, que estén en contacto con sus tierras, de acuerdo al ambiente y a sus intereses y cuyos maestros fueran de su misma raza (...)" (FOERSTER; MONTECINO, 1988, p. 262). El contar con un profesorado de la misma raza, aseguraba en perspectiva de los profesores mapuche, el habla originaria en la formación del estudiantado y con ello su proyección en las nuevas generaciones, para que "no dejen las costumbres, la lengua, como lo hace el inglés, el alemán que viene acá: siguen hablando y siguen con sus costumbres de inglés o alemán” (1988, p. 176).

$\mathrm{Al}$ respecto, la Comisión para el estudio de la enseñanza indígena, presidida por José Inalaf, conformada en el Congreso de Profesores Radicales durante el año 1939, planteó como una de sus conclusiones que "la enseñanza del escolar araucano sea impartida en los primeros años tanto en castellano como en araucano, de acuerdo con un programa especial" (1988, p. 263).

Para asegurar una efectiva enseñanza desde el bilingüismo la misma comisión estableció entre sus declaraciones finales la necesidad de disponer de un cuerpo de profesores mapuche, proponiendo la creación de una institución formadora de profesores específica para mapuches:

6. Que sea obligación moral de los profesores mapuches recién egresados de las escuelas Normales servir como maestros en las escuelas ubicadas en las reducciones indígenas durante tres años, y que el Estado les asigne un sobresueldo como estímulo [...]. 8. Que se funde una Escuela Normal para Indígenas de uno y otro sexo, sin perjuicio de que intertanto se dupliquen las becas que en conformidad al reglamento se destinan para ello [...] (1988, p. 263).

Otra organización conformada porjóvenes intelectuales y educadores mapuche expresaba, el año 1943, la necesidad de tomar medidas educativas tendientes a rectificar los errores de la historia (en referencia a la historia escolar) "que han contribuido a menoscabar la dignidad del pueblo aborigen", entre ellas la creación de cursos especiales dirigidos a mapuches para que "sirvan de maestros en las escuelas donde existan reducciones, ya que profesores que no conocen el dialecto y la idiosincrasia del aborigen jamás podrán adaptarse a ellos" (1988, p. 272).

Similares propósitos se pueden visualizar en la declaración emitida por la primera agrupación de profesores mapuche que se organiza hacia 1946, a propósito de la realización del Primer Congreso Pedagógico de Maestros Araucanos, donde declaran velar por la defensa cultural "y los intereses sociales de los niños de la raza araucana” (1988, p. 264).

Las diversas voces del profesorado de la época, confluyen en la aspiración por encontrar en la escuela chilena un espacio de enseñanza para la cultura y lengua originaria, en igual consideración que la lengua castellana. Articulan un discurso en torno a las contradicciones y diferencias respecto del mundo no-mapuche, e insistieron en la idea de acercar la enseñanza a las comunidades y la necesidad de implementar una educación especial para indígenas, que considerara el conocimiento y lengua mapuche, y ello solo podía realizarse con profesores pertenecientes al propio pueblo originario.

Los profesores mapuche sabían de los maltratos y desarraigo cultural que provocaba la escuela entre los jóvenes de la etnia, por lo que disponer de un profesorado proveniente de la propia cultura originaria se visualizaba como una posibilidad de aminorar los niveles de discriminación que la escuela chilena profería al estudiantado mapuche. El testimonio de la profesora Zenobia Quintremil refrenda esta situación:

Desde mi infancia, cuando asistía a la escuela rural mixta en Almagro, a una legua de mi casa, empezó a germinar en mi mente la idea de estudiar para llegar a convertirme en una profesora y poder influir, algún día, 
en el cambio de rumbos que debe imprimirse a la enseñanza. No puedo decir que tales ideas eran nítidas... pero constituian una reacción natural en contra del sistema de enseñanza, de la conducta de la Directora, que imponía castigos propios de la era del coloniaje y hacía distingos entre "chilenos" y "araucanos"... donde quiera que estuve... pude observar y sentir los efectos de lo que bien puedo denominar "discriminación racial" cuyo menor efecto consiste en considerar al araucano como un ser inferior [...] (1988, p. 268).

Es por ello que esta misma profesora, levantó con fuerza su voz para demandar una formación del profesorado acorde a las características culturales del contexto:

[...] en la enseñanza es indispensable ir a la formación de un profesorado especialmente adiestrado...con verdadera vocación y en lo social, dándoles idéntico tratamiento a chilenos y a los mapuches, así como iguales oportunidades para realizar cualquier clase de empresas (1988, p. 269).

En definitiva, se trata de una demanda transformadora para la época que además de ilustrar la larga data de la reivindicación por una formación docente en la lengua indígena, puede presentarse como un antecedente histórico de la actual educación intercultural bilingüe en Chile.

\section{Discusión}

La demanda por un profesorado con formación en lalenguay cultura mapucheconstituye una reivindicación educativa de larga data en el contexto interétnico mapuche/no mapuche. Si bien se trata de requerimientos realizados por agentes educadores con motivaciones y pertenencia cultural distinta, en lo sustantivo estas demandas abogan por la presencia de un cuerpo docente con habilitación profesional para desarrollar procesos pedagógicos con pertinencia cultural.
Desde las voces del profesorado mapuche, la demanda por un profesorado conocedor de la lengua y la cultura, representa una forma de resistencia a la situación de dominación e imposición cultural que significó el arribo a sus tierras del sistema de enseñanza y principios formativos chileno-occidentales. Estos profesores, formados en el sistema educativo chileno, si bien reconocían fortalezas en el acceso a la educación chilena, tenían como motivación para su demanda educativa aminorar la arbitrariedad cultural del programa de formación hegemónico y promover, por el contrario, una enseñanza que otorgue cabida a los patrones culturales de la sociedad originaria, para preservar espacios de producción y reproducción de la matriz cultural propia en el sistema educativo.

Por su parte, las actuales demandas de formación del profesorado para el contexto educativo interétnico, se levantan desde voces docentes vinculadas étnica y culturalmente a la chilenidad, pero con una experiencia laboral en el territorio mapuche que les permite reconocer la pertinencia educativa de una formación en la lengua y cultura indígena. Aquello da cuenta del saber acumulado que poseen las comunidades de profesores en su experiencia profesional en contexto de pueblo originario, que les permite nutrir con contenidos pertinentes la formación de profesores para el desempeño en este tipo de contextos educativos, pero que los procesos de renovación curricular de formación de profesores no han considerado en sus diseños, insistiendo más bien en itinerarios formativos homogeneizadores.

Cabe destacar la impronta transformadora de estas demandas, en la medida que involucran una formación para el trabajo con saberes culturales originarios desde el dominio de la lengua, ámbitos que posicionan a la cultura indígena, en este caso la cultura mapuche, como parte constituyente del currículo de formación inicial (TURRA; FERRARA; VILLENA, 2013) e implica romper con una formación uniforme, para avanzar hacia el diseño de currículos que diversifiquen sus procesos de formación en consideración a la diversidad cultural y 
lingüistica en que se desarrolla la acción educativa (FERRADA; TURRA; VILLENA, 2013).

Resulta evidente que, tanto ayer como hoy, la formación del profesorado en Chile al desconsiderar las demandas de formación en la lengua y cultura indígena, muestran la distancia que existe entre los diseños de política pública que formaliza la formación docente $\mathrm{y}$ los requerimientos y necesidades reales de los profesores en los escenarios educativos interétnicos. En la actualidad, esta omisión resulta preocupante en tanto se encuentra en contradicción con las normativas jurídicas que buscan revalorizar los saberes culturales originarios, a través del sistema educativo (Ley Indígena, Convenio 169), y más aún con el reciente decreto ministerial que incorporan la Lengua Indígena como sector de aprendizaje en la educación básica chilena (Decreto 280), situación que no se traduce en una formación de profesores que efectivamente puedan dar cuenta de las exigencias de desempeño profesional que el propio estado intenciona.

En esta perspectiva, la política educativa para pueblos originarios no puede persistir en desoír esta histórica demanda de las comunidades docentes situadas, pues cualquier pretensión de transformación educativa que busque fortalecer la cultura y lengua indígena, así como el desarrollo de procesos pedagógicos fundados en la interculturalidad, requiere de un cuerpo de profesores habilitados profesionalmente para desempeñarse con propiedad en el logro de estos propósitos. Esto significa asumir la experiencia socioeducativa que indica que cualquier cambio o transformación educativa se juega en la sala de clases, con un relevante protagonismo del profesorado (FULLAN, 2002; AGUERRONDO, 2004; VAILLANT, 2005).

\section{Conclusiones}

Las características culturales y lingüísticas propias que presenta el estudiantado mapuche que asiste al sistema escolar en el contexto territorial de la Araucanía representaron en el pasado y en la actualidad la razón fundamental para sostener una demanda por un profesorado formado en la lengua y cultura originaria. Se trata de demandas que pueden caracterizarse como transformadoras en la medida que reconocen legitimidad a la cultura y lengua mapuche en el sistema escolar y proyectan una incorporación, en consecuencia, en el currículo de formación inicial de profesores. Desde su dimensión histórica se agrega además, su búsqueda por introducir el bilingüismo en la enseñanza y con ello no solo se pretende dar cuenta de la diferencia sociolingüistica del estudiantado mapuche, sino que constituye un primer antecedente en la reivindicación por una educación intercultural en Chile.

Desde la diversidad lingüística propia de los contextos de pueblos originarios en contacto interétnico, las demandas educativas resultan necesarias e incuestionables, también desde los consensos internacionales que promueven la incorporación de las lenguas originarias en el sistema educativo para garantizar los derechos lingüísticos y la igualdad educativa para estas comunidades.

Finalmente, la persistencia en el tiempo de esta demanda educativa habla de una recurrencia en las prácticas de diseño de programas de formación del profesorado, cual es la desconsideración de las voces de las comunidades de profesores desde los contextos de desempeño. 


\section{Referencias}

AGUERRONDO, Inés. Los desafíos de la política educativa relativos a las reformas de la formación docente. In: VAILLANT, Denise; ROSS, Cecilia (Ed.). Maestros en América Latina: nuevas perspectivas sobre su formación y desempeño. Santiago de Chile: PREAL - CINDE, 2004. p. 97-142.

AUBERT, Adriana; DUQUE, Elena; FISAS, Monserrat; VALLS, Rosa. Dialogar y transformar. Barcelona: Graó, 2004.

BENGOA, José. Historia del pueblo Mapuche (Siglo XIX y XX). Santiago: LOM, 2000.

BESALÚ, Xavier. Diversidad cultural y educación. Madrid: Síntesis, 2002.

BUSTOS, Antonio. Enseñar en la escuela rural aprendiendo a hacerlo. Evolución de la identidad profesional en las aulas multigrado. Profesorado, Granada, v. 11, n. 3, p. 0, 2007.

CANALES, Pedro. Peyepeyen. Escuelas chilenas en contextos mapuche, integración y resistencia, 1860-1950. Última Década, Viña del Mar, n. 9, p. 3- 15. 1998.

CARLOSAMA, Jesús. Formación y capacitación de docentes ingas en etnoeducación, Santiago, Putumayo, Colombia, 2001. (Tesina de Maestría) - Universidad Mayor San Simón, Cochabamba, 2001.

CATRIQUIR, Desiderio; DURÁN, Teresa. Ad engu Kimûn: principios educativos Mapuche, bases para un currículum educacional. In: SEMINARIO DE EDUCACIÓN INTERCULTURAL BILINGÜE, 1., 1997, Temuco. Primer... Temuco: Universidad Católica de Temuco, 1997.

CATRIQUIR, Desiderio; DURÁN, Teresa. Gûnerzuamgerpunmew epuxokiñ rakizuam. Abriendo cauces de con-versación entre pensamientos. Anthropos, Barcelona, n. 207, p. 97-108, 2007.

CRESWELL, John W. Research design: qualitative, quantitative, and mixed methods approaches. Los Angeles: Sage, 2009.

DÍAZ-BARRIGA, Angel. Diversidad cultural y currículum. ¿Es factible una articulación? Pensamiento Educativo, Santiago de Chile, n. 37, p. $52-63$, 2005.

FERRADA, Donatila. Tratamiento de datos cualitativos en la metodología comunicativa crítica: una propuesta para la discusión. Temuco: [s. n.], 2011. Documento no publicado.

FERRADA, Donatila; TURRA, Omar. Las comunidades de profesores como agentes legítimos en la construcción curricular para la formación inicial. Revista de Pedagogía Crítica Paulo Freire, v. 11, n. 11, p. 207-217, jul. 2012.

FERRADA, Donatila; TURRA, Omar; VILLENA, Alicia. Currículum transformador de formación inicial para profesores en contextos de vulnerabilidad social. Cadernos de Pesquisa, São Paulo, v. 43, n. 149, p. 642-661, maio/ago. 2013.

FOERSTER, Rolf; MONTECINO, Sonia. Organizaciones, líderes y contiendas mapuches (1900-1970). Santiago: CEM, 1988.

FULLAN, Michael. Las fuerzas del cambio: explorando las profundidades de la reforma educativa. Madrid: Akal, 2002.

GÓMEZ, Aitor; DÍEZ-PALOMAR, Javier. Metodología comunicativa crítica: transformaciones y cambios en el siglo XXI. Teoría de la Educación, Salamanca, v. 10, n. 3, p. 103-118, 2009.

GÓMEZ, Aitor; HOLFORD, John. Contribuciones al éxito educativo desde la metodología comunicativa. Revista Educación y Pedagogía, Medelin, v. 22 , n. 56, p. $21-29,2010$

GÓMEZ, Aitor; RACIONERO, Sandra. El paradigma comunicativo crítico. Universitas Tarraconensis. Revista de Ciències de l'Educació, Barcelona, v. 32, n. 3, p. 117-129, 2008.

GÓMEZ, Jesus et al. Metodología comunicativa crítica. Barcelona: El Roure, 2006.

GONZÁLEZ-VALLINAS, Paula; OTERINO, David; SAN FABIÁN, José. Factores asociados a la formación permanente del profesorado de educación secundaria en Asturias. Profesorado, Granada, v. 11, n. 1, p. 1-13, 2007.

HERNÁNDEZ, Crescenciano. La formación profesional del docente bilingüe de la jefatura de Cuicatlán (desde la perspectiva intercultural), 2008. (Tesina de Maestría) - Universidad Pedagógica Nacional, México, 2008.

HERNÁNDEZ, Roberto; FERNÁNDEZ, Carlos; BAPTISTA, Pilar. Metodología de la investigación. México, DF.: Mc Graw Hill, 2010.

LÓPEZ, Eustacio. Formación de docentes indígenas en la Universidad Pedagógica Nacional. Contextos y líneas de reflexión sobre la identidad profesional y ética, 2005. (Tesina de Maestría) - Universidad Pedagógica Nacional, México, 2005. 
LÓPEZ, Luis; SICHRE, Inge. La educación en áreas indígenas de América Latina: balances y perspectivas. In: HERNAíZ, Ignacio (Org.). Educación en la diversidad: experiencias y desafíos en la educación intercultural bilingüe. Buenos Aires: IIPE: Unesco, 2004. p. 121-149.

MANZO, Luis; WESTERHOUT, Carlos. Propuesta metodológica en educación intercultural para contextos urbanos. Cuadernos Interculturales, Viña del Mar, v. 1, n. 1, p. 3-47, 2003.

MARIMÁN, Pablo. Demanda por educación en el movimiento mapuche en Chile 1910-1995. In: BELLO, Álvaro et al. Pueblos indígenas: educación y desarrollo. Temuco: Instituto de Estudios Indígenas, 1997. p. 130-200.

MARTÍNEZ, Christian. Políticas colonizadoras de Chile en el siglo XIX: la ilusión modernizadora, Araucanía 1813-1913. Estudios Sociales, Santiago de Chile, n. 69, p. 79-91, 1991

PADRÓS, María et al. Contrasting scientific knowledge from the lifeworld: the dialogic inclusion contract. Qualitative Inquiry, v. 17, n. 3, p. 304-312, 2011.

PINTO, Jorge. Misioneros en la Araucanía, 1600-1900: un capítulo de historia fronteriza en Chile. Bogotá: CELAM, 1990.

PINTO, Jorge. Crisis económica y expansión territorial: la ocupación de la Araucania en la segunda mitad del siglo XIX. Estudios Sociales, Santiago de Chile, n. 72, p. 85-126, 1992.

PINTO, Jorge. De la inclusión a la exclusión: la formación del estado, la nación y el pueblo mapuche. Santiago: Instituto de Estudios Avanzados. Universidad de Santiago de Chile, 2000.

QUINTRIQUEO, Segundo. Implicancias de un modelo curricular monocultural en contexto mapuche. Temuco: Universidad Católica de Temuco, 2010.

RAYÓN, Ignacio. La formación docente de los profesores indígenas de la región cuicaleca en el contexto del movimiento pedagógico: trayectorias profesionales, 2004. (Tesina de Maestría) - Universidad Pedagógica Nacional, México, 2004.

ROBALINO, Magaly. Formación docente y TICs: logros, tensiones y desafíos estudio de 17 experiencias en América Latina. Santiago de Chile: Unesco, 2005.

SEPÚLVEDA, Gastón. ¿Qué es aprendizaje expansivo? Ponencia presentada al Encuentro Nacional de Educación y Patrimonio. Santiago de Chile: CPEIP, 2001.

SERRANO, Sol. De escuelas indígenas sin pueblos a pueblos sin escuelas indígenas: la educación en la Araucanía en el siglo XIX. Historia, n. 29, p. 423-474, 1995-96.

TORRES, Jurjo. Diversidad cultural y contenidos escolares. Revista de Educación, Madrid, n. 345, p. 83-110, 2008.

TURRA, Omar; FERRADA, Donatila; VILLENA, Alicia. La especificidad del contexto indígena como requerimiento para la formación inicial del profesorado. Estudios Pedagógicos, Valdivia, v. 39, n. 2, p. 7-26, 2013.

VAILLANT, Denise. Formación de docentes en América Latina: re-inventando el modelo tradicional. Barcelona: Octaedro, 2005.

VEZUB, Lea. La formación y el desarrollo profesional docente frente a los nuevos desafíos de la escolaridad. Profesorado, Granada, v. 11, n. 1, p. 5-24, 2007.

WILLIAMSON, Guillermo. ¿Educación multicultural, educación intercultural bilingüe, educación indígena o educación intercultural? Cuadernos Interculturales, Valparaiso, v. 2, n. 3, p. 23-34. 2004.

Recibido en: 30.10.2014

Aprobado en: 13.05.2015

Omar Turra Díaz es profesor e investigador asociado de la Facultad de Educación y Humanidades, Universidad del Bio Bio.

Donatila Ferrada Torres es investigadora y profesora de la Facultad de Educación, Universidad Católica del Maule. 